\title{
The Effect of Hermanson's Spatial Dielectric Function on the Density of Impurity States in a Gallium Arsenide Quantum Dot of Rectangular Cross-Section
}

\author{
Leonard Machuka, Hannigton Odhiambo Oyoko \\ Department of Physics, Pwani University, Kilifi, Kenya \\ Email address: \\ muyemachuka@gmail.com (L. Machuka),h.oyoko@pu.ac.ke (H. O. Oyoko)
}

\section{To cite this article:}

Leonard Machuka, Hannigton Odhiamb Oyoko. The Effect of Hermanson's Spatial Dielectric Function on the Density of Impurity States in a Gallium Arsenide Quantum Dot of Rectangular Cross-Section. International Journal of Applied Mathematics and Theoretical Physics. Vol. 4, No. 3, 2018, pp. 73-77. doi: 10.11648/j.ijamtp.20180403.12

Received: August 19, 2018; Accepted: September 17, 2018; Published: October 17, 2018

\begin{abstract}
We have carried out a theoretical study of the effect of Hermanson's spatial dielectric function on the density of impurity states (DOIS) for a shallow hydrogenic donor impurity located in the center of a Gallium Arsenide (GaAs) Quantum Well Dot (QWD) of rectangular cross-section. The density of impurity states (DOIS) of an unscreened (hydrogenic) donor impurity was calculated and compared with that of the screened (non-hydrogenic) donor impurity for the same system. Our calculations were carried out using a trial wave function within the effective mass approximation. Our calculations have been carried out with finite barriers. In this study, we first calculated the ground state binding energies of both hydrogenic and nonhydrogenic donor impurity for different dot sizes. The donor binding energies in the two regimes are then used to compute the DOIS. The results show that for both hydrogenic and non-hydrogenic donor impurities, the DOIS sharply rises to a peak, and then decreases almost exponentially with increase in binding energy. The results also show that the DOIS obtained for the nonhydrogenic donor impurities is markedly enhanced over that for purely hydrogenic donor impurities in which a dielectric constant is employed in the potential. In fact, the enhanced DOIS is observed throughout the range of values for binding energy considered. To a good extend there is good agreement between our results and those reported in the literature. It is therefore, important that the effect of the spatial dielectric function should be considered when designing optoelectronic devices.
\end{abstract}

Keywords: Density of Impurity States, Hydrogenic Donor, Non Hydrogenic Donor, Effective Mass Approximation, Spatial Dielectric Function, Quantum Dot, Donor Impurity

\section{Introduction}

The physics of shallow-donor impurity states in semiconductor quantum-dot structures is an interesting area of research owing to the ability to manipulate their electronic and optical properties [1-2]. Much research has been carried out on the superlattices of a GaAs layer sandwiched between $\mathrm{Ga}_{-\mathrm{x}} \mathrm{Al}_{\mathrm{x}} \mathrm{As}$ barriers because of its direct bandgap. Owing to this, it emits and absorbs light efficiently making it applicable in a wide range of semiconductor devices e.g. solar cells, solid state laser photodetectors, etc [3]. When impurities such as hydrogenic and non-hydrogenic donors are introduced into semiconductors; they affect carrier transport and optical properties of such semiconductors. This is because; they introduce bound states in the forbidden gap of such materials [4]. A number of theoretical and experimental studies have been carried out on various effects of these donor impurities in semiconductor nanostructures such as quantum wells (QW), quantum well wires (QWW) and quantum dots (QD), Oyoko [5] and others [6-11]. Elabsy [12] has reported results for the calculation of the effect of hydrostatic stress on the donor impurity binding energy. Oyoko [13] has carried out studies on the effect of uniaxial stress on the density of impurity states [DOIS] of shallow hydrogenic donor impurities in GaAs quantum wells. The results show a variation of DOIS with binding energy having two peaks. Increased stress delays the onset of variation of DOIS with donor impurity binding energy. Duque et al [1314] have calculated the density of impurity states (DOIS) for a homogeneous distribution of acceptor impurities within the low-dimensional heterostructure. They have reported that for 
dimensions of the system in which the length is much larger than the radius we obtain two well-defined peaks, associated with acceptors either at the on-centre position or at the edge position in the low-dimensional system. More recently, Abarna et al [15] have reported the effect of dielectric screening on the binding energy of a hydrogenic donor in a Square Quantum well composed of vacuum/GaAs/GaAlAs, as a function of well width and Aluminum composition. Their results showed that decrease in the size of the well increases the binding energy, reaching a peak value at a particular well width. Thereafter, the binding energy decreases for further decrease in well width. It is further observed that the peak value of the hydrogenic donor binding energy increases when the effect of Dielectric screening function is considered.

In this research work, we have carried out a theoretical study of the effect of Hermanson's spatial dielectric function [16] on the DOIS of shallow donors in a GaAs quantum well dot (QWD) of rectangular cross-section This present treatment is distinct from earlier reported findings in the sense that we have considering the effect of screening on the DOIS. This paper is organized as follows: in Section 2 we present a theoretical framework on which the work is based. In Section 3 we present results and discussions and finally Section 4 we present the conclusions derived from the results of this work.

$$
\begin{aligned}
& H=\frac{-\hbar^{2}}{2 m^{*}}\left[\frac{\partial^{2}}{\partial x^{2}}+\frac{\partial^{2}}{\partial y^{2}}+\frac{\partial^{2}}{\partial z^{2}}\right]-\frac{e^{2}}{4 \pi \varepsilon_{0}} \frac{1}{\left(x^{2}+y^{2}+z^{2}\right)}+V_{B}(x, y, z) \\
& H=\frac{-\hbar^{2}}{2 m^{*}}\left[\frac{\partial^{2}}{\partial x^{2}}+\frac{\partial^{2}}{\partial y^{2}}+\frac{\partial^{2}}{\partial z^{2}}\right]-\frac{e^{2}}{4 \pi \varepsilon(x, y, z)} \frac{1}{\left(x^{2}+y^{2}+z^{2}\right)}+V_{B}(x, y, z)
\end{aligned}
$$

Where;

$\mathrm{m}^{*}=0.067 \mathrm{~m}_{\mathrm{o}}$, is the effective mass of an electron.

The confining potential $\mathrm{V}_{\mathrm{B}}$ is given by;

$$
V_{B}(x, y, z)=\left\{\begin{array}{cc}
0 & |x| \leq \frac{L_{x}}{2},|y| \leq \frac{L_{y}}{2}|z| \leq \frac{L_{z}}{2} \\
\infty & \text { elsewhere }
\end{array}\right.
$$

We have used the spatial screening dielectric function suggested by Hermanson's which has the form,

$$
\frac{1}{\varepsilon(r)}=\frac{1}{\varepsilon_{0}}+\left(1-\frac{1}{\varepsilon_{0}}\right) e^{-c r}
$$

Where $r=\sqrt{\left(x^{2}+y^{2}+z^{2}\right)}$

Our value of $c=0.8 a u^{-1}$, was obtained by Oyoko and

\section{Theoretical Framework}

We have used a trial wave function, which is modified by an additional term that represents the hydrogenic impurity as given in equation (1).

$$
\psi(x, y, z)=N \cos \alpha x \cos \beta y \cos \lambda z
$$

Where, $\mathrm{N}$ represents the normalization constant and $\delta$ is the variational parameter. The cosine functions confine the donor impurity inside the QWD. $\alpha, \beta$, and $\lambda$ are constants.

We have considered the ground state with $n=1$, such that,

$$
\alpha=\frac{\pi}{L_{x}}, \beta=\frac{\pi}{L_{y}}, \lambda=\frac{\pi}{L_{z}}
$$

$L_{x}, L_{y}$, and $L_{z}$ represent the dimensions of the length of the quantum dot in the $x, y$, and $z$ directions respectively. For our case we have taken,

$$
L_{x}=L_{y}+L_{z}=100 \mathrm{~nm}
$$

The three-dimensional Hamiltonian used for this problem is given by equations (2) and (3) for hydrogenic and nonhydrogenic donor impurity cases respectively.
Csavinszky [17]

It is clear from equation (5) that when $r \rightarrow 0$, $\varepsilon(x, y, z) \rightarrow 1$ and as

$$
r \rightarrow \infty, \varepsilon(x, y, z) \rightarrow \varepsilon_{0}=12.56
$$

The Schrodinger equation is solved by variational method with $\langle H\rangle$ given by,

$$
\langle H\rangle=\langle T\rangle+\langle V\rangle
$$

Where $\langle T\rangle$ and $\langle V\rangle$ are the expectation values of the kinetic and potential energy respectively.

The expectation of the kinetic energy is given by equation 


$$
\begin{gathered}
\langle T\rangle=\frac{-\hbar^{2} N^{2}}{2 m^{*}}\left\{\alpha^{2}+\beta^{2}+\lambda^{2}-\delta^{2}\right\} \int_{0}^{\frac{L_{x}}{2}} \cos ^{2} \alpha x d x \int_{0}^{\frac{L_{y}}{2}} \cos ^{2} \beta y d y \int_{0}^{\frac{L_{z}}{2}} \cos ^{2} \lambda z \exp \left[-2 \delta\left(x^{2}+y^{2}+z^{2}\right)^{\frac{1}{2}}\right] d z+ \\
\frac{\hbar^{2} N^{2}}{2 m^{*}} \int_{0}^{\frac{L_{x}}{2}} \cos ^{2} \alpha x d x \int_{0}^{\frac{L_{y}}{2}} \cos ^{2} \beta y d y \int_{0}^{\frac{L_{z}}{2}} \cos ^{2} \lambda z \exp \left[-2 \delta\left(x^{2}+y^{2}+z^{2}\right)^{\frac{1}{2}}\right] \\
-\frac{\hbar^{2} N^{2} \alpha \delta}{2 m^{*}} \int_{0}^{\frac{L_{x}}{2}} x \cos \alpha x \sin \alpha x d x \int_{0}^{\frac{L_{y}}{2}} \cos ^{2} \beta y d y \int_{0}^{\frac{1}{2}} \frac{\left(x^{2}+z^{2}\right)^{\frac{L_{z}}{2}} \cos ^{2} \lambda z \exp \left[-2 \delta\left(x^{2}+y^{2}+z^{2}\right)^{\frac{1}{2}}\right]}{\left.2 y^{2}\right)^{\frac{1}{2}}} d z \\
-\frac{\hbar^{2} N^{2} \beta \delta}{2 m^{*}} \int_{0}^{\frac{L_{x}}{2}} \cos ^{2} \alpha x d x \int_{0}^{\frac{L_{y}}{2}} y \cos \beta y \sin \beta y d y \int_{0}^{\frac{L_{z}}{2}} \frac{\cos ^{2} \lambda z \exp \left[-2 \delta\left(x^{2}+y^{2}+z^{2}\right)^{\frac{1}{2}}\right]}{2 m^{*}} \int_{0}^{\frac{L_{x}}{2}} \cos ^{2} \alpha x d x \int_{0}^{\frac{L_{y}}{2}} \cos ^{2} \beta y d y \int_{0}^{\frac{L_{z}}{2}} \frac{\left(x^{2}+y^{2}+z^{2}\right)^{\frac{1}{2}}}{\left(\hbar^{2}\right.} \frac{\left(x^{2}+y^{2}+z^{2}\right)^{\frac{1}{2}}}{\hbar^{2}} d z \sin \lambda z \exp \left[-2 \delta\left(x^{2}+y^{2}+z^{2}\right)^{\frac{1}{2}}\right]
\end{gathered}
$$

The potential energy $\langle V\rangle$ is calculated as given in equation (8).

$$
\langle V\rangle=-\frac{e^{2} N^{2}}{4 \pi \varepsilon_{0}} \int_{0}^{\frac{L_{x}}{2}} \cos ^{2} \alpha x d x \int_{0}^{\frac{L_{y}}{2}} \cos ^{2} \beta y d y \int_{0}^{\frac{L_{z}}{2}} \frac{\cos ^{2} \lambda z \exp \left[-2 \delta\left(x^{2}+y^{2}+z^{2}\right)^{\frac{1}{2}}\right]}{\left(x^{2}+y^{2}+z^{2}\right)^{\frac{1}{2}}} d z
$$

Where, $\mathrm{N}^{2}$ is the square of the normalization constant which is given as,

$$
N^{2}=\left\{\int_{0}^{\frac{L_{x}}{2}} \cos ^{2} \alpha x d x \int_{0}^{\frac{L_{y}}{2}} \cos ^{2} \beta y d y \int_{0}^{\frac{L_{z}}{2}} \cos ^{2} \lambda z \exp \left[-2 \delta\left(x^{2}+y^{2}+z^{2}\right)^{\frac{1}{2}}\right] d z\right\}^{-1}
$$

The expectation value of the total energy $\langle H\rangle$ for the hydrogenic and non-hydrogenic donor is computed and then minimized with respect to the variational parameter subject to the condition in equation (10).

$$
\frac{d\langle H\rangle}{d \delta}=0,
$$

The value of $\delta$ obtained from equation (10) is plucked back into equations (7) and (8) which are computed and added to give us the minimum total energy $\langle E\rangle_{\min }$.

Considering the free particle which is a donor impurity with no potential applied to it, the free particle ground state wave function is given by equation (11).

$$
\psi(x, y, z)=N \cos \alpha x \cos \beta y \cos \lambda z
$$

The Hamiltonian, for the free particle is given by equation (12);

$$
H_{\text {free }}=\frac{-\hbar^{2}}{2 m^{*}}\left[\frac{\partial^{2}}{\partial x^{2}}+\frac{\partial^{2}}{\partial y^{2}}+\frac{\partial^{2}}{\partial z^{2}}\right]
$$

Using equations (11) and (12), the free particle energy $\langle E\rangle_{\text {free }}$ will be, 


$$
\langle E\rangle_{\text {free }}=\frac{-\hbar^{2} N^{2}}{2 m^{*}}\left\{\alpha^{2}+\beta^{2}+\lambda^{2}\right\} \int_{0}^{\frac{L_{x}}{2}} \cos ^{2} \alpha x d x \int_{0}^{\frac{L_{y}}{2}} \cos ^{2} \beta y d y \int_{0}^{\frac{L_{z}}{2}} \cos ^{2} \lambda z d z
$$

This simplifies to,

$$
\langle E\rangle_{\text {free }}=\frac{-\hbar^{2}}{2 m^{*}}\left\{\alpha^{2}+\beta^{2}+\lambda^{2}\right\}
$$

We then obtain the binding energy for the donor impurity by subtracting the minimum energy from the free particle energy,

$$
\langle E\rangle_{b}=\langle E\rangle_{\text {free }}-\langle E\rangle_{\min }
$$

The density of impurity states (DOIS) is obtained from the binding energy using equation (16),

$$
g\left(E_{b}\right)=\frac{1}{L_{x} L_{y} L_{z}} \int \frac{\nabla L}{\nabla\left|E_{b}\right|}
$$

\section{Results and Discussions}

Figure 1 shows the variation of the DOIS as a function of binding energy for a hydrogenic donor impurity.

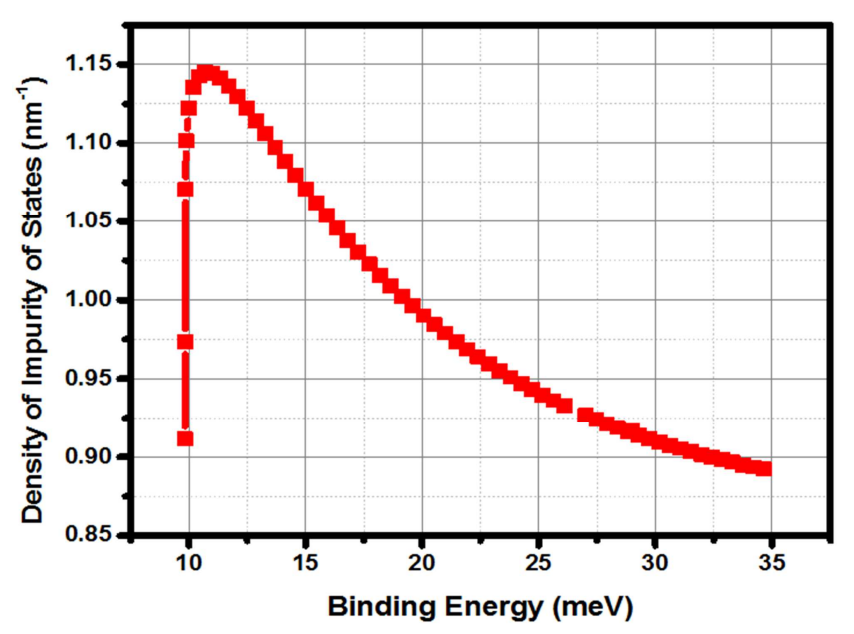

Figure 1. Density impurity states as a function of binding energy for the hydrogenic donor impurity with $\varepsilon=12.56$. The DOIS quickly rises to a peak value and then decreases with nicrease in binding energy.

From Figure 1, we observe that the density of impurity states starts at about $0.92 / \mathrm{nm}$ and sharply increases to a peak of $1.15 / \mathrm{nm}$ at about $10.7 \mathrm{meV}$. There is then a sharp and almost exponential decrease in DOIS with increase in binding energy to a value of about $0.87 / \mathrm{nm}$ at around $35 \mathrm{meV}$. The differences in DOIS more significantly show up below $25 \mathrm{meV}$ and show more deviations as the binding energy decreases up to about the peak value. This behavior is consistent with previous research findings which have reported an important feature that is a peak in DOIS at lower binding energies that comes from the contribution of impurities near the axial edge of the quantum well dot.
In figure 2 we present a plot of the DOIS as a function of the binding energy for the non-hydrogenic donor impurity with the dielectric constant replaced by the spatial dielectric function given by equation (5).

A similar trend in variation of DOIS as a function of binding energy is observed as in the case of the hydrogenic donor impurity. The onset of DOIS is about $0.92 / \mathrm{nm}$ rising sharply to a peak of about $1.19 / \mathrm{nm}$ at about $13.5 \mathrm{meV}$. The DOIS then reduces almost exponentially to about $0.97 / \mathrm{nm}$ at $38 \mathrm{meV}$. -

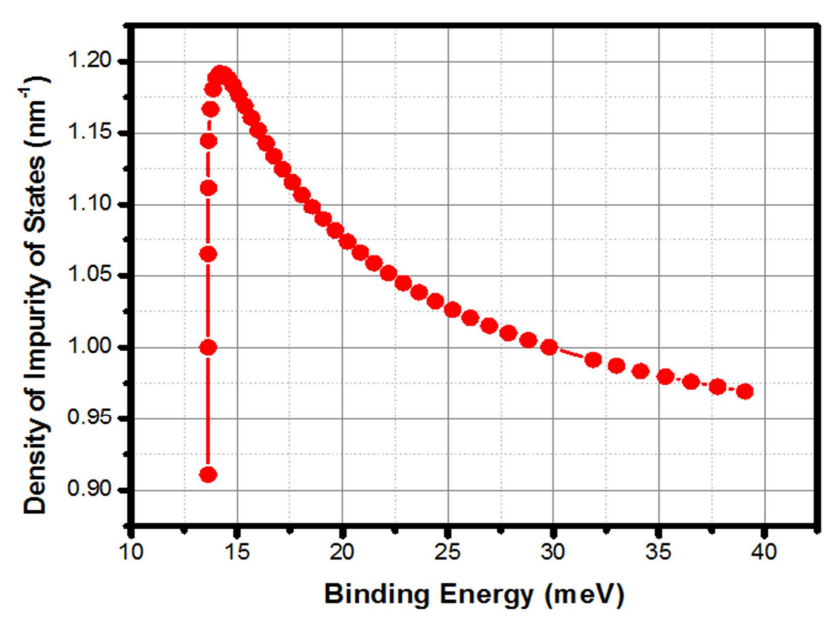

Figure 2. Density of impurity states as a function of binding energy for a non-hydrogenic donor impurity. Notice the DOIS sharply rises to a peak and then decreases almost exponentially with increase in binding energy.

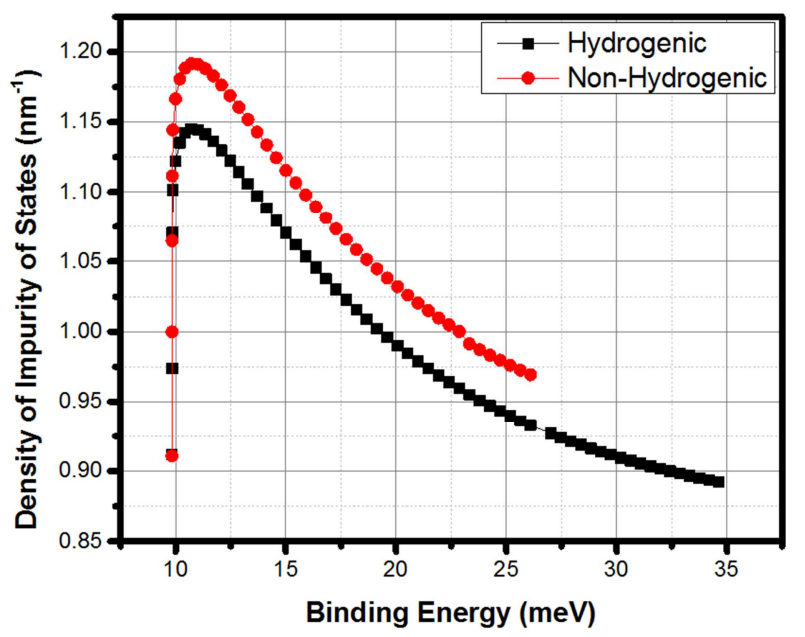

Figure 3. A plot of the density of impurity states as a function of binding energy of both hydrogenic and non-hydrogenic donors. The DOIS for the non-hydrogenic donor impurity is larger than that of the hydrogenic donor right for values of binding energies above $12 \mathrm{meV}$.

Figure 3 shows a plot of the DOIS for both hydrogenic and non-hydrogenic donor impurities together. It is clear from the results displayed that DOIS for non-hydrogenic donor impurities is much larger than that for the hydrogenic donor 
right from the onset of DOIS through to their peaks and, in fact, the former becomes larger as the binding energy increases. This is reasonable because as the donor impurity approaches the donor ion core, it is less screened by the dielectric function since the dielectric function approaches the dielectric constant while as the donor moves further away from its parent ion, the screening becomes more pronounced.

These results have been compared with previously reported findings by Ribeiro and Latge [18] for the spherical and cuboid geometry of GaAs QD. In their case, the effect of electric field and dielectric function was not included. The results of the impurity density of exhibited a similar shape, where both structures had the same volume. It was clear from the results that the two impurity densities of states also had the same important single peaked feature at low binding energies.

\section{Conclusion}

We have performed a theoretical calculation related to the effect of Hermanson's spatial dielectric function on the density of impurity states (DOIS) in $G a A s / G a_{1}-x A l_{x} A s$ QD of rectangular cross-section using a variational procedure within the effective mass approximation. Our results show that;

1. The density of impurity states DOIS starts at a particular value and sharply increases to a maximum value at low binding energy. This is then followed by a sharp and almost exponential drop in DOIS to an almost flat value at higher binding energy.

2. The DOIS for the non-hydrogenic donor impurity was much larger than that of the hydrogenic donor impurity throughout the range of binding energies considered.

3. The difference in the DOIS is much more pronounced at the peaks and then is maintained throughout the range of values for binding energy considered

The effect of the dielectric function (screening) is that it enhances the density of impurity states above in the in the non-hydrogenic donor impurity over the hydrogenic (unscreened) donor impurities. These results should add to our knowledge and understanding of experimental results related to optical phenomena associated with shallow donor impurities in quantum well dots of different geometry.

\section{Acknowledgements}

The authors are very grateful and wish to thank Professor Odhiambo Amolo of Technical University of Kenya for the helpful discussions, Ms Winnie Achieng and Dr Manyali of Masinde Muliro university for their assistance in developing the script for this work.

\section{References}

[1] Bastard, G. (1981). Hydrogenic impurity states in a quantum well: A simple model. Physical Review B, 24 (8), 4714.

[2] Khordad, R., Sedehi, H. R., \& Bahramiyan, H. (2018). Effects of impurity and cross-sectional shape on entropy of quantum wires. Journal of Computational Electronics, 17 (2), 551-561.

[3] Bugajski, M., \& Nakwaski, W. (2017). Physics of semiconductor lasers. Elsevier.

[4] Harrison, P., \& Valavanis, A. (2016). Quantum wells, wires and dots: theoretical and computational physics of semiconductor nanostructures. John Wiley \& Sons Mroziewicz, B.

[5] Oyoko, H. O., Porras-Montenegro, N., López, S. Y., \& Duque, C. A. (2007, December). Physica Status Solidi (C) Current Topics in Solid State Physics. In conference.

[6] Tiutiunnyk, A., Akimov, V., Tulupenko, V., Mora-Ramos, M. E., Kasapoglu, E., Ungan, F.... \& Duque, C. A. (2016). Electronic structure and optical properties of triangular $\mathrm{GaAs} / \mathrm{AlGaAs}$ quantum dots: Exciton and impurity states. Physica B: Condensed Matter, 484, 95-108.

[7] Masselink, W. T., Chang, Y. C., \& Morkoc, H. (1984). Binding energies of acceptors in GaAs-Al x Ga1- x As quantum wells. Journal of Vacuum Science \& Technology B: Microelectronics Processing and Phenomena, 2 (3), 376-382.

[8] Csavinszky, P., \& Elabsy, A. M. (1985). Dielectric response to a donor ion in a $\mathrm{Ga} 1-\mathrm{x} \mathrm{Al} \times \mathrm{x}$ As-GaAs-Ga 1- $\mathrm{x} \mathrm{Al} \mathrm{x} \mathrm{As}$ quantum well of infinite depth. Physical Review B, 32 (10), 6498.

[9] Brown, J. W., \& Spector, H. N. (1986). Hydrogen impurities in quantum well wires. Journal of applied physics, 59 (4), 1179-1186.

[10] Csavinszky, P., \& Oyoko, H. (1991). Binding energy of onaxis hydrogenic and non hydrogenic donors in a $\mathrm{GaAs} / \mathrm{Ga} 1-$ $\mathrm{x}$ Al $\mathrm{x}$ As quantum-well wire of circular cross section. Physical Review B, 43 (11), 9262.

[11] Elabsy, A. M. (1993). Hydrostatic pressure dependence of binding energies for donors in quantum well heterostructures. Physica Scripta, 48 (3), 376.

[12] Montes, A., Duque, C. A., \& Porras-Montenegro, N. (1998). Density of shallow-donor impurity states in rectangular cross section GaAs quantum-well wires under applied electric field. Journal of Physics: Condensed Matter, 10 (24), 5351.

[13] Oyoko, H. O., Duque, C. A., \& Porras-Montenegro, N. (2001). Uniaxial stress dependence of the binding energy of shallow donor impurities in $\mathrm{GaAs}-(\mathrm{Ga}, \mathrm{Al})$ As quantum dots. Journal of Applied Physics, 90 (2), 819-823.

[14] Duque, C. A., Porras-Montenegro, N., Barticevic, Z., Pacheco, M., \& Oliveira, L. E. (2006). Effects of applied magnetic fields and hydrostatic pressure on the optical transitions in self-assembled InAs/GaAs quantum dots. Journal of Physics: Condensed Matter, 18 (6), 1877.

[15] Abarna, R., Anitha, A., \& Arulmozhi, M. (2017). Effects of Dielectric Screening Function and Image Charges on Hydrogenic Donor Binding Energy in a Surface Quantum Well. Journal of Physical Science, 28 (1), 73.

[16] J. Hermanson, Phys. Rev. 150, 660 (1996).

[17] P. Csavinszky and H. Oyoko. Phys. Rev. B 43, 9262 Published 15 April 1991.

[18] F. J. Ribeiro, A. Latge, Phys. Rev. B 50 (1994) 4913. 